\title{
THE ASYMPTOTIC BEHAVIOUR OF FRACTIONAL LATTICE SYSTEMS WITH VARIABLE DELAY
}

\author{
Linfang Liu ${ }^{1}$, Tomás Caraballo ${ }^{2}$, Peter E. Kloeden ${ }^{3}$
}

\begin{abstract}
The existence and uniqueness of global solutions for a fractional functional differential equation is established. The asymptotic behaviour of a lattice system with a fractional substantial time derivative and variable time delays is investigated. The existence of a global attracting set is established. It is shown to be a singleton set under a certain condition on the Lipschitz constant.
\end{abstract}

MSC 2010: Primary 26A33; Secondary 37K40, 37L6, 39B72

Key Words and Phrases: fractional substantial derivative, fractional lattice systems, variable delay, Leray-Schauder theorem, global attracting sets

\section{Introduction}

In this paper, we study the global existence and the long time behaviour of solutions for a fractional delay lattice system of the form

$$
\left\{\begin{array}{l}
D_{s}^{\alpha} u_{i}(t)-\left(u_{i-1}(t)-2 u_{i}(t)+u_{i+1}(t)\right)+\lambda u_{i}(t)+f_{i}\left(t, u_{i t}\right)=0, t \geq \tau \\
u_{i}(t)=\phi_{i}(t-\tau), \forall t \in[\tau-h, \tau]
\end{array}\right.
$$

where $\lambda \in \mathbb{R}, 0<\alpha<1, i \in \mathbb{Z}$ and the Caputo fractional substantial derivative $D_{s}^{\alpha}$ is defined as

$D_{s}^{\mu} f(x)=D_{s}^{m}\left[I_{s}^{\nu} f(x)\right], \nu=m-\mu, m$ is the smallest integer which exceeds $\mu$, where

$$
I_{s}^{\nu} f(x)=\frac{1}{\Gamma(\nu)} \int_{0}^{x}(x-\tau)^{\nu-1} e^{-\beta(x-\tau)} f(\tau) d \tau, \quad \nu>0
$$

is the fractional substantial integral [5, 7] and $\Gamma$ is the Gamma function. $\beta$ is a positive constant and

(C) Year Diogenes Co., Sofia

pp. $\mathrm{xxx}-\mathrm{xxx}, \mathrm{DOI}$ : 


$$
D_{s}^{m}=\left(\frac{\partial}{\partial x}+\beta\right)^{m}=(D+\beta)^{m}=(D+\beta)(D+\beta) \ldots(D+\beta) .
$$

The concept of fractional substantial derivative was introduced in 2006 by Friedrich et al. [8] to consider retardation effects in Kramers-FokkerPlanck type equations, where these are expressed mathematically by a fractional substantial derivative, which is more appropriate to characterize nonlocal coupling in time and space than the classic fractional derivative. The fractional substantial derivative is widely applied in physics, such as long jump of Lévy flights, anomalous diffusion, turbulence research, FokkerPlanck equations, forward and backward fractional Feynman-Kac equations etc [13, 6]. Moreover, the fractional substantial diffusion model is useful in applications to geophysics [1] as well as finance [4. In finance, where the tempered stable process models price fluctuation which displays semiheavy tails, resembling a power law behavior at moderate time scales, but finally converging to a Gaussian at large time scales. See [8, 3] for more information about the application of fractional substantial derivative, [10] and related literatures for the classic fractional calculus.

A main objective is to analyze the existence of solutions of fractional functional differential equations with the Caputo fractional substantial derivative, since there has been little published on this topic or on fractional lattice systems with delay.

First we use Leray-Schauder's fixed point theorem to prove the existence of local solutions for a fractional functional differential equation with $\alpha \in$ $(0,1)$. Then, by a priori estimates, we prove that the local solution is actually a global one. Our assumption here on delay term $f\left(t, u_{t}\right)$ is weaker than many available references. Finally, we apply this result to a fractional lattice system with variable delay, i.e., system (1.1), and obtain the existence and uniqueness of global solutions, as well as global attracting sets. Our result also applies to fractional lattice systems with distributed delay.

The paper is structured as follows. Notation, some basic definitions and preliminary results are included in the next section. Additionally, Section 2 is also devoted to the global existence and uniqueness of solutions for an abstract fractional functional differential equation (2.1) in a separable Hilbert space. This theoretical result is applied to a fractional delay lattice system in Section 3. A priori estimates are obtained in Section 4 and used in Section 5 to establish the main result of this paper, namely, the existence of global attracting set while, which becomes a singleton under appropriate conditions. 


\section{Preliminaries}

Let $X$ be a separable Hilbert space with norm $\|\cdot\|$ and inner product $(\cdot, \cdot)$. For a fixed $\tau \in \mathbb{R}, \mathcal{C}_{T}:=C([\tau-h, T] ; X), h>0, T \geq 0$, be the Banach space of continuous functions $u:[\tau-h, T] \rightarrow X$, endowed with the norm $\|u\|_{\mathcal{C}_{T}}=\sup _{\tau-h \leq s \leq T}\|u(s)\|$. For any $u \in \mathcal{C}_{T}$ and $t \in[\tau, T]$, we denote by $u_{t}$ the element of $\overline{\mathcal{C}_{0}}=C([-h, 0] ; X)$ defined by $u_{t}(\theta)=u(t+\theta), \theta \in[-h, 0]$.

The dual coupling between $X$ and its dual $X^{*}$ is denoted by $\langle\cdot, \cdot\rangle$. Since $X$ is a Hilbert space, by the Riesz representation theorem, $X^{*}$ is isomorphic to $X$. So the dual coupling $\langle\cdot, \cdot\rangle$ is the inner product in $X$.

Let $X_{w}$ be the space $X$ endowed with the weak topology. We consider the space $C_{0, w}=C\left([-h, 0] ; X_{w}\right)$ and say that $u_{t}^{n} \rightarrow u_{t}$ in $C_{0, w}$ if for any $\theta \in[-h, 0]$ with $u^{n}\left(t+\theta_{n}\right) \rightarrow u(t+\theta)$ in $X_{w}$ for all $\theta_{n} \rightarrow \theta$.

We say that the function $f:[0, \infty) \times \mathcal{C}_{0} \rightarrow X$ is weakly continuous in bounded sets for each $t \in[0, \infty)$, if $u^{n} \rightarrow u$ in $C_{0, w}$, and $\left\|u^{n}\right\|_{\mathcal{C}_{0}} \leq M$ for all $n$ imply that $f\left(t_{n}, u_{t}^{n}\right) \rightarrow f\left(t, u_{t}\right)$ in $X_{w}$. Similar concepts were introduced in 22 to establish the existence of solutions for a delay differential equation in a Banach space.

We consider the global existence theorems for the following delay differential equations with Caputo fractional substantial time derivative in the separable Hilbert space $X$,

$$
\begin{cases}D_{s}^{\alpha} u(t)=f\left(t, u_{t}\right), & t \geq \tau \\ u(t)=\phi(t-\tau), & \forall t \in[\tau-h, \tau]\end{cases}
$$

where the nonlinear term $f$ is weakly continuous in bounded sets.

Definition 2.1. A function $u \in \mathcal{C}_{T}$ is called a solution of the initial value problem (2.1) if $u(t)=\phi(t-\tau)$ for $t \in[\tau-h, \tau]$ with $\phi \in \mathcal{C}_{0}$, and, for $t \in[\tau, T], u(t)$ satisfies the integral equation

$$
u(t)=\phi(0) e^{-\beta(t-\tau)}+\frac{1}{\Gamma(\alpha)} \int_{\tau}^{t}(t-r)^{\alpha-1} e^{-\beta(t-r)} f\left(r, u_{r}\right) d r, \quad t \in[\tau, T] .
$$

Hereafter, we assume that:

$\left(\mathbf{H}_{\mathbf{1}}\right)$ The mapping $f:[\tau, \infty) \times \mathcal{C}_{0} \rightarrow \mathbb{R}$ is continuous, and there exists $M_{1}>0$ such that

$$
\|f(t, \varphi)-f(t, \psi)\| \leq M_{1}\|\varphi-\psi\|_{\mathcal{C}_{0}} \quad \text { for all } \varphi, \psi \in \mathcal{C}_{0} \text { and } t \in[\tau, \infty) .
$$

$\left(\mathbf{H}_{2}\right)$ The function $f(t, 0)$ is $L^{1+\frac{1}{\alpha}}$ locally integrable, and there exists $G>0$ such that

$$
\int_{\tau}^{t}(t-r)^{\alpha-1} e^{-\beta(t-r)}\|f(r, 0)\|^{2} d r \leq G, \quad \forall t \geq \tau .
$$


Now for any $\phi \in \mathcal{C}_{0}$, we define an operator $\mathcal{S}_{\phi}: \mathcal{C}_{T} \rightarrow \mathcal{C}_{T}$ by $\mathcal{S}_{\phi} u(t):=\left\{\begin{array}{l}\phi(t-\tau), \quad t \in[\tau-h, \tau], \\ \phi(0) e^{-\beta(t-\tau)}+\frac{1}{\Gamma(\alpha)} \int_{\tau}^{t}(t-r)^{\alpha-1} e^{-\beta(t-r)} f\left(r, u_{r}\right) d r, t \in[\tau, T] .\end{array}\right.$

We can prove that the operator $\mathcal{S}_{\phi}$ is well-defined on $\mathcal{C}_{T}$ by a similar method as Lemma 2.1, so the details are omitted.

Since $X$ is a separable space, there exists a sequence such that the subset $\left\{e_{1}, e_{2}, \cdots, e_{n}, \cdots\right\}$ is dense in $X$, and by the Gram-Schmidt procedure, we can obtain an orthonormal subset of $X$, still denoted by $\left\{e_{1}, e_{2}, \cdots, e_{n}, \cdots\right\}$. Taking $X_{n}=\left\{e_{1}, e_{2}, \cdots, e_{n}\right\}$, and $P_{n}: X \rightarrow X_{n}$ an orthonormal projector, defined as $P_{n} u=u_{n}$, and $P_{n} \phi=\phi_{n}$, for every $n$, we can introduce the mapping $S_{n, \phi}: \mathcal{C}_{T} \rightarrow \mathcal{C}_{T}$ as, for any $\phi_{n}$,

$S_{n, \phi} u_{n}(t):=\left\{\begin{array}{l}\phi_{n}(t-\tau), \quad t \in[\tau-h, \tau], \\ \phi_{n}(0) e^{-\beta(t-\tau)}+\frac{1}{\Gamma(\alpha)} \int_{\tau}^{t}(t-r)^{\alpha-1} e^{-\beta(t-r)} P_{n} f\left(r, u_{n, r}\right) d r, t \in[\tau, T] .\end{array}\right.$

We will need the following auxiliary theorem.

Theorem 2.1. (Leray-Schauder's fixed point theorem [9, 12]) Let $T$ be a continuous and compact mapping of a Banach space $X$, such that the set $\{x \in X: x=\lambda T x$, for some $0 \leq \lambda \leq 1\}$ is bounded. Then $T$ possesses a fixed point.

For any positive constant $\rho$, denote by

$$
A(\rho):=\left\{u_{n} \in \mathcal{C}_{T}: u_{n}(t)=\phi_{n}(t-h), t \in[\tau-h, \tau] \text {, and } \sup _{t \in[\tau, T]}\left\|u_{n}(t)\right\| \leq \rho\right\} .
$$

Lemma 2.1. For any $\phi \in \mathcal{C}_{0}$, the operator $S_{n, \phi}$ maps the bounded subset $A(\rho) \subset \mathcal{C}_{T}$ into another bounded set $A\left(\rho^{\prime}\right) \subset \mathcal{C}_{T}$.

P r o o f. For our purpose, it is enough to show that for $\forall \rho>0$, there exists a positive constant $\rho^{\prime}$ such that for each $u_{n} \in A(\rho)$ we have $\sup _{t \in[\tau, T]}\left\|S_{n, \phi} u_{n}(t)\right\| \leq \rho^{\prime}$, where $A(\rho)$ is defined in $(2.3)$. Let $u_{n} \in A(\rho)$ be arbitrary. From the definition of $S_{n, \phi}$ as in $(2.2)$, we have for all $t \in[\tau, T]$,

$$
\left\|S_{n, \phi} u_{n}(t)\right\|^{2} \leq 2\left\|\phi_{n}(0)\right\|^{2}+\frac{2 \beta^{-\alpha}}{\Gamma(\alpha)} \int_{\tau}^{t}(t-r)^{(\alpha-1)} e^{-\beta(t-r)}\left\|f\left(r, u_{n, r}\right)\right\|^{2} d r
$$


By $\left(\mathbf{H}_{\mathbf{1}}\right)$ and $\left(\mathbf{H}_{\mathbf{2}}\right)$, we derive

$$
\left\|f\left(r, u_{n, r}\right)\right\|^{2}=\left\|f\left(r, u_{n, r}\right)-f(r, 0)+f(r, 0)\right\|^{2} \leq 2 M_{1}^{2}\left\|u_{n, r}\right\|_{\mathcal{C}_{0}}^{2}+2\|f(r, 0)\|^{2},
$$

which together with $(2.4)$ and the Gronwall lemma implies that

$$
\left\|S_{n, \phi} u_{n}(t)\right\|_{\mathcal{C}_{T}}^{2} \leq 2\|\phi\|_{\mathcal{C}_{0}}^{2}+\frac{2 \beta^{-\alpha} G}{\Gamma(\alpha)}+2 M_{1}^{2} \beta^{-2 \alpha} \rho^{2}:=\rho^{\prime 2}<\infty, \forall t \in[\tau, T] .
$$

Hence, $S_{n, \phi}$ maps the bounded set $A(\rho)$ into another bounded set $A\left(\rho^{\prime}\right)$.

Lemma 2.2. For any $\phi \in \mathcal{C}_{0}$, the mapping $S_{n, \phi}$ is continuous and completely continuous.

P r o o f. Step 1: First, we verify that $S_{n, \phi} A(\rho)$ is relatively compact. Since $X_{n}$ has finite dimension $n$, it suffices to prove that the family of continuous functions $\left\{S_{n, \phi} u_{n}: u_{n} \in A(\rho)\right\}$ is uniformly bounded and is a family of equicontinuous functions of $\mathcal{C}_{T}$ on $[\tau, T]$. As proved in Lemma 2.1. for any $u_{n} \in A(\rho)$, we find $\left\|S_{n, \phi} u_{n}(t)\right\|_{\mathcal{C}_{T}} \leq \rho^{\prime}$, which implies that $\left\{S_{n, \phi} u_{n}: u_{n} \in A(\rho)\right\}$ is uniformly bounded.

On the other hand, for any $t_{1}, t_{2} \in[\tau, T]$ with $t_{1}<t_{2}$, we have

$$
\left\|S_{n, \phi} u\left(t_{1}\right)-S_{n, \phi} u\left(t_{2}\right)\right\| \leq e^{\beta \tau}\left\|\phi_{n}(0)\right\|\left|e^{-\beta t_{1}}-e^{-\beta t_{2}}\right|+A_{1}+A_{2} .
$$

By $\left(\mathbf{H}_{2}\right)$ and the Hölder inequality, $A_{1}$ can be bounded by

$$
\begin{aligned}
A_{1} & =\frac{1}{\Gamma(\alpha)}\left\|\int_{\tau}^{t_{1}}\left[\left(t_{1}-r\right)^{\alpha-1} e^{-\beta\left(t_{1}-r\right)}-\left(t_{2}-r\right)^{\alpha-1} e^{-\beta\left(t_{2}-r\right)}\right] f\left(r, u_{n, r}\right) d r\right\| \\
& \leq \frac{M_{1}^{\prime} \rho^{\prime}}{\Gamma(\alpha+1)}\left[\left(t_{1}-\tau\right)^{\alpha}-\left(t_{2}-\tau\right)^{\alpha}+\left(t_{2}-t_{1}\right)^{\alpha}\right]+\frac{1}{\Gamma(\alpha)}\left(\int_{\tau}^{t_{1}}\left(t_{1}-r\right)^{\alpha-1}\right. \\
& \left.\left.-\left(t_{2}-r\right)^{\alpha-1}\right)^{p} d r\right)^{\frac{1}{p}} \times\left(\int_{\tau}^{t_{1}}\|f(r, 0)\|^{q} d r\right)^{\frac{1}{q}}+\frac{M_{1} \rho^{\prime}}{\beta^{\alpha}}\left(1-e^{-\beta\left(t_{2}-t_{1}\right)}\right) \\
& +\frac{\left(1-e^{-\beta\left(t_{2}-t_{1}\right)}\right)}{\sqrt{\Gamma(\alpha)} \beta^{\alpha}}\left(\int_{\tau}^{t_{1}}\left(t_{1}-r\right)^{\alpha-1} e^{-\beta\left(t_{1}-r\right)}\|f(r, 0)\|^{2} d r\right)^{\frac{1}{2}} .
\end{aligned}
$$

Again, thanks to the Hölder inequality,

$$
\begin{aligned}
A_{2} & =\frac{1}{\Gamma(\alpha)}\left\|\int_{t_{1}}^{t_{2}}\left(t_{2}-r\right)^{\alpha-1} e^{-\beta\left(t_{2}-r\right)} f\left(r, u_{n, r}\right) d r\right\| \\
& \leq \frac{M_{1} \rho\left(t_{2}-t_{1}\right)^{\alpha}}{\Gamma(\alpha+1)}+\frac{\left(t_{2}-t_{1}\right)^{\frac{\alpha}{2}}}{\sqrt{\alpha} \Gamma(\alpha)} \cdot\left(\int_{t_{1}}^{t_{2}}\left(t_{2}-r\right)^{\alpha-1} e^{-\beta\left(t_{2}-r\right)} \|\left. f(r, 0)\right|^{2} d r\right)^{\frac{1}{2}} .
\end{aligned}
$$

From (2.5), (2.6) and (2.7), the right-hand side of (2.5) tends to zero as $t_{1} \rightarrow t_{2}$. Therefore $\left\{S_{n, \phi} u_{n}: u_{n} \in A(\rho)\right\}$ is equicontinuous on $[\tau, T]$, and therefore $S_{n, \phi} A(\rho)$ is relatively compact in $X_{n}$. 
Step 2: Now we show that $S_{n, \phi}$ is continuous on $\mathcal{C}_{T}$. Let $u_{n}^{i} \in \mathcal{C}_{T}$ be a sequence such that $u_{n}^{i} \rightarrow u$ in $\mathcal{C}_{T}$ as $i \rightarrow \infty$. We can prove that, for any $t \in[\tau, T]$, as $i$ goes to $\infty$,

$$
\left\|u_{n, t}^{i}-u_{t}\right\|_{\mathcal{C}_{T}}=\sup _{\theta \in[-h, 0]}\left\|u_{n, t}^{i}(t+\theta)-u(t+\theta)\right\| \leq \sup _{t \in[\tau, T]}\left\|u_{n, t}^{i}(t)-u(t)\right\| \rightarrow 0 .
$$

Then from Eq. (2.2), (H4) and the Hölder inequality, we have for all $t \in[\tau, T]$

$$
\begin{aligned}
& \left\|S_{n, \phi} u_{n}^{i}(t)-S_{n, \phi} u(t)\right\|^{2} \\
& =\frac{1}{\Gamma^{2}(\alpha)}\left\|\int_{\tau}^{t}(t-r)^{\alpha-1} e^{-\beta(t-r)}\left(P_{n} f\left(r, u_{n, r}^{i}\right)-P_{n} f\left(r, u_{r}\right)\right) d r\right\|^{2} \\
& \leq \frac{M_{1}^{2} \beta^{-\alpha}}{\Gamma(\alpha)} \int_{\tau}^{t}(t-r)^{(\alpha-1)} e^{-\beta(t-r)}\left\|u_{n, r}^{i}-u_{r}\right\|_{\mathcal{C}_{0}}^{2} d r .
\end{aligned}
$$

Hence,

$$
\left\|S_{n, \phi} u_{n}^{i}(t)-S_{n, \phi} u(t)\right\|^{2} \leq M_{1}^{2} \beta^{-2 \alpha}\left\|u_{n}^{i}-u\right\|_{\mathcal{C}_{T}}^{2} \rightarrow 0 \text { as } i \rightarrow \infty .
$$

Therefore the operator $S_{n, \phi}$ is continuous on $\mathcal{C}_{T}$. By the arbitrariness of $\rho$ and Lemma 2.1, we have that $S_{n, \phi} u_{n}^{i}$ is uniformly bounded on $\mathcal{C}_{T}$, while Step 1 implies that $S_{n, \phi} u_{n}^{i}$ is a family of equi-continuous functions. Therefore, by the Arzelà-Ascoli theorem, we obtain that $S_{n, \phi} u_{n}^{i} \rightarrow S_{n, \phi} u$ in $\mathcal{C}_{T}$. And hence, from Lemma 2.1, Step 1, Step 2 and the Arzelà-Ascoli theorem, we obtain that $S_{n, \phi}: C\left([\tau-h, T] ; X_{n}\right) \rightarrow C\left([\tau-h, T] ; X_{n}\right)$ is continuous and completely continuous.

Lemma 2.3. Assume that $(\mathbf{H 1})-(\mathbf{H 2})$ hold true, then for any $\phi \in \mathcal{C}_{0}$, the fractional functional differential equation (2.1) has at least one local solution in $X_{n}$.

$\mathrm{P}$ r o o f. We use the Leray-Schauder fixed point theorem to prove this conclusion, i.e., there exists an open set $\mathcal{O} \subset C\left([\tau-h, T] ; X_{n}\right)$ with $u_{n} \neq$ $\lambda S_{n, \phi} u_{n}$ for $\lambda \in(0,1)$ and $u_{n} \in \partial \mathcal{O}$.

We need to prove that if $u_{n}=\lambda S_{n, \phi} u_{n}$ with $\lambda \in(0,1)$ on $\overline{\mathcal{O}}$, then $u_{n} \in \operatorname{int} \mathcal{O}$.

Let $u_{n} \in C\left([\tau-h, T] ; X_{n}\right)$ and $u_{n}=\lambda S_{n, \phi} u_{n}$ for some $0<\lambda<1$. Then for every $t \in[\tau, T]$,

$$
u_{n}(t)=\lambda\left\{\phi_{n}(0) e^{-\beta(t-\tau)}+\frac{1}{\Gamma(\alpha)} \int_{\tau}^{t}(t-r)^{\alpha-1} e^{-\beta(t-r)} P_{n} f\left(r, u_{n, r}\right) d r\right\}
$$


From $(\mathbf{H 1})-(\mathbf{H 2})$ and the Hölder inequality with $p=1+\alpha$ and $q=1+\frac{1}{\alpha}$, we find for all $t+\theta \geq \tau$,

$$
\begin{aligned}
& \left\|u_{n}(t+\theta)\right\| \\
& =\lambda\left\|\phi_{n}(0) e^{-\beta(t+\theta-\tau)}+\frac{1}{\Gamma(\alpha)} \int_{\tau}^{t+\theta}(t+\theta-r)^{\alpha-1} e^{-\beta(t+\theta-r)} P_{n} f\left(r, u_{n, r}\right) d r\right\| \\
& \leq\left\|\phi_{n}(0) e^{-\beta(t+\theta-\tau)}\right\|+\frac{\beta^{-\alpha} G}{\Gamma(\alpha)}+T_{0}\left(\int_{\tau}^{t+\theta} e^{-\frac{q \beta(t+\theta-r)}{2}}\left\|u_{r}-v_{r}\right\|_{\mathcal{C}_{0}}^{q} d r\right)^{\frac{1}{q}}
\end{aligned}
$$

where $0<G(t+\theta)=\frac{\beta^{-\alpha}}{\Gamma(\alpha)}\left(\int_{\tau}^{t+\theta}(t+\theta-r)^{\alpha-1} e^{-\beta(t+\theta-r)}\|f(r, 0)\|^{2} d r\right)$, and $T_{0}=\frac{M_{1} \Gamma\left(\alpha^{2}\right)^{\frac{1}{1+\alpha}}}{\left(\frac{p \beta}{2}\right)^{\frac{\alpha^{2}}{1+\alpha}} \Gamma(\alpha)}$.

Note that if $\tau-h \leq t \leq \tau$, then

$$
\left\|u_{n}(t)\right\|=\left\|\phi_{n}(t-\tau)\right\| \leq e^{-\frac{q \beta(t+2 \theta-2 \tau)}{2}}\left\|\phi_{n}\right\|_{\mathcal{C}_{0}} .
$$

Then for any $t \geq \tau$,

$$
e^{\frac{q \beta t}{2}}\left\|u_{n, t}\right\|_{\mathcal{C}_{0}}^{q} \leq C_{*} e^{-\frac{q \beta(t-2 \tau)}{2}}\left\|\phi_{n}\right\|_{\mathcal{C}_{0}}^{q}+G_{1} e^{\frac{q \beta t}{2}}+T_{1} \int_{\tau}^{t} e^{\frac{q \beta r}{2}}\left\|u_{n, r}\right\|_{\mathcal{C}_{0}}^{q} d r
$$

where $C_{*}=3^{q} e^{q \beta h}, G_{1}=\frac{\beta^{-q \alpha}}{\Gamma^{q}(\alpha)} 3^{q-1} G^{q}$ and $T_{1}=3^{q-1} T_{0}^{q} e^{\frac{q \beta h}{2}}$.

Applying Gronwall's inequality, we find for all $t \geq \tau$,

$$
\left\|u_{n, t}\right\|_{\mathcal{C}_{0}}^{q} \leq C e^{\left(T_{1}-\frac{q \beta}{2}\right)(t-\tau)}+C .
$$

Thus, there exists a positive constant $B^{*}$ such that $\left\|u_{n, t}\right\|_{\mathcal{C}_{0}} \leq B^{*}$ for all $t \in[\tau, T]$. Set

$$
\mathcal{O}=\left\{u_{n} \in C\left([\tau-h, T] ; X_{n}\right):\left\|u_{n}\right\|_{\mathcal{C}_{T}} \leq B^{*}+1\right\} .
$$

Note that $S_{n, \phi}: \overline{\mathcal{O}} \rightarrow C\left([\tau-h, T] ; X_{n}\right)$ is continuous and completely continuous. From the choice of $\mathcal{O}$, there is no $u_{n} \in \partial \mathcal{O}$ such that $u_{n}=\lambda S_{n, \phi}\left(u_{n}\right)$ for $\lambda \in(0,1)$, on the other hand $0 \in \mathcal{O}$ is obvious. Therefore, as a consequence of Theorem 2.1, we deduce that $S_{n, \phi}$ has a fixed point $u_{n}$ in $\mathcal{O}$, which is a local solution of $(2.1)$ in $C\left([\tau-h, T] ; X_{n}\right)$.

Lemma 2.4. Under the assumptions of Lemma 2.3, for any $\phi \in C_{0}$, problem 2.1 has a local solution in $C([\tau-h, T] ; X)$.

P r o o f. In Lemma 2.3, we have proved the existence of local solutions of problem (2.1) in $C\left([\tau-h, T] ; X_{n}\right)$. By taking the limit, we prove the 
existence of local solutions of problem (2.1) in $C([\tau-h, T] ; X)$. For this, we form the approximating equations:

$$
\begin{cases}D_{s}^{\alpha} u_{n}(t)=P_{n} f\left(t, u_{n, t}\right), & t \geq \tau \\ u_{n}(t)=\phi_{n}(t-\tau), & \forall t \in[\tau-h, \tau] .\end{cases}
$$

Similarly as in Lemma 2.3. there exists a solution $u_{n}$ to problem 2.9 on $[\tau, T]$ such that

$$
\sup _{t \in[\tau, T]}\left\|u_{n}(t)\right\| \leq C
$$

Since $X$ is a separable Hilbert space, from 2.10 we can conclude that for any $t \in[\tau, T],\left\{u_{n}(t)\right\}_{n=1}^{\infty}$ is weakly convergent to $u(t)$ in $X$. Moreover for any $t_{m}, t \in[\tau-h, \tau]$ with $t_{m} \rightarrow t$, we have when $n, m$ go to $\infty$,

$$
\left\|u_{n}\left(t_{m}\right)-u(t)\right\| \leq\left\|\phi_{n}\left(t_{m}-\tau\right)-\phi\left(t_{m}-\tau\right)\right\|+\left\|\phi\left(t_{m}-\tau\right)-\phi(t-\tau)\right\| \rightarrow 0 .
$$

On the other hand, for any $t_{m}, t \in[\tau, T]$ with $t_{m} \rightarrow t$, and any $v \in X^{*}$, we have as $n, m$ go to $\infty$,

$$
\left|\left\langle u_{n}\left(t_{m}\right)-u(t), v\right\rangle\right| \leq\left|\left\langle u_{n}\left(t_{m}\right)-u\left(t_{m}\right), v\right\rangle\right|+\left|\left\langle u\left(t_{m}\right)-u(t), v\right\rangle\right| \rightarrow 0 .
$$

Therefore, $\left\{u_{n, t}\right\}_{n=1}^{\infty}$ is weakly convergent to $u_{t}$ in $\mathcal{C}_{0}$.

Now we prove that $u(\cdot)$ is a solution of $(2.1)$. For this we need to pass to the limit in the following integral

$$
u_{n}(t):=\left\{\begin{array}{l}
\phi_{n}(t-\tau), \quad t \in[\tau-h, \tau], \\
\phi_{n}(0) e^{-\beta(t-\tau)}+\frac{1}{\Gamma(\alpha)} \int_{\tau}^{t}(t-r)^{\alpha-1} e^{-\beta(t-r)} P_{n} f\left(r, u_{n, r}\right) d r, t \in[\tau, T] .
\end{array}\right.
$$

Since $f$ is Lipschitz continuous, for any $t \in[\tau, T]$, we obtain

$$
f\left(t, u_{n, t}\right) \rightarrow f\left(t, u_{t}\right), \text { weakly in } X .
$$

Using the Riesz representation theorem, we obtain that for any $v \in X^{*}$, there exists a unique $w \in X$ such that $\langle u, v\rangle=(u, w)$ for all $u \in X$, and therefore

$$
\begin{aligned}
& \left|\left\langle P_{n} f\left(t, u_{n, t}\right), v\right\rangle-\left\langle f\left(t, u_{n, t}\right), v\right\rangle\right| \\
& =\left|\left(P_{n} f\left(t, u_{n, t}\right)-f\left(t, u_{n, t}\right), w\right)\right| \\
& =\left|\left(f\left(t, u_{n, t}\right),\left(I-P_{n}\right) w\right)\right| \leq C\left\|\left(I-P_{n}\right) w\right\| \rightarrow 0, n \rightarrow \infty .
\end{aligned}
$$

Then by 2.12)-(2.13) and Lebesgue's dominated convergence theorem we obtain for any $v \in X^{*}$,

$\left\langle\int_{\tau}^{t}(t-r)^{\alpha-1} e^{-\beta(t-r)} P_{n} f\left(r, u_{n, r}\right) d r, v\right\rangle \rightarrow\left\langle\int_{\tau}^{t}(t-r)^{\alpha-1} e^{-\beta(t-r)} f\left(r, u_{r}\right) d r, v\right\rangle$.

Hence, 2.11 and 2.14 imply that, for any $v \in X^{*}$, 


$$
\langle u(t), v\rangle=\left\langle u(\tau) e^{-\beta(t-\tau)}, v\right\rangle+\frac{1}{\Gamma(\alpha)}\left\langle\int_{\tau}^{t}(t-r)^{\alpha-1} e^{-\beta(t-r)} f\left(r, u_{r}\right) d r, v\right\rangle,
$$

which means that

$$
u(t)=u(\tau) e^{-\beta(t-\tau)}+\int_{\tau}^{t}(t-r)^{\alpha-1} e^{-\beta(t-r)} f\left(r, u_{r}\right) d r, \text { for all } t \in[\tau, T] .
$$

TheOREM 2.2. (Global existence and uniqueness and continuous dependence on the initial values of solutions of Caputo substantial fractional differential equation) Suppose that $\left(\mathbf{H}_{\mathbf{1}}\right)$ and $\left(\mathbf{H}_{\mathbf{2}}\right)$ hold. Then,

(i) for any $\phi \in \mathcal{C}_{0}$, the initial value problem (2.1) with the initial condition $u(t)=\phi(t-\tau), t \in[\tau-h, \tau]$, has a unique global solution $u(\cdot, \phi)$ on the whole interval $[\tau-h, \infty)$.

(ii) on any bounded time interval $[\tau, T]$, where $T>0$, the solution $u(\cdot, \phi)$ depends continuously on $\phi$, i.e.,

$$
\lim _{\phi \rightarrow \varphi} \sup \|u(t, \phi)-v(t, \varphi)\|_{\mathcal{C}_{T}}=0
$$

P r o o f. Part (i): From Lemmas 2.4, we know that 2.1) has a local solution on $[\tau-h, T]$. So we need to prove that the local solution is a global one. Namely, we prove that the local solutions will not blow up on any bounded interval. By a similar procedure as we used in Lemma 2.3 , we can prove that for any solution $u(t)$, we have for all $t \geq \tau$,

$$
\left\|u_{t}\right\|_{\mathcal{C}_{0}}^{q} \leq C e^{\left(T_{1}-\frac{q \beta}{2}\right)(t-\tau)}+C,
$$

which implies that the local solution $u(t)$ will not blow up on any finite interval $[\tau, T]$. Then it is actually a global solution on $[\tau-h, \infty)$.

Finally, we show that the global solution is unique. Let $u(t), v(t)$ be solutions of (2.1) with the same initial value $\phi$. Then for $t \in[\tau, T]$,

$$
\|u(t)-v(t)\|=\left\|\frac{1}{\Gamma(\alpha)} \int_{\tau}^{t}(t-r)^{\alpha-1} e^{-\beta(t-r)}\left(f\left(r, u_{r}\right)-f\left(r, v_{r}\right)\right) d r\right\| .
$$

Replacing $t$ by $t+\theta$ in (2.15), note that $\|u(t+\theta)-v(t+\theta)\|=0$ when $t+\theta \leq \tau$ with $\theta \in[-h, 0]$. On the other hand, taking $p=1+\alpha, q=1+\frac{1}{\alpha}$, then by Hölder's inequality, we have, for $t+\theta \geq \tau$,

$$
\|u(t+\theta)-v(t+\theta)\| \leq T_{0}\left(\int_{\tau}^{t+\theta} e^{-\frac{q \beta(t+\theta-r)}{2}}\left\|u_{r}-v_{r}\right\|_{\mathcal{C}_{0}}^{q} d r\right)^{\frac{1}{q}}
$$

which means (here $T_{0}$ is defined in (2.8)) 


$$
e^{\frac{q \beta t}{2}}\left\|u_{t}-v_{t}\right\|_{\mathcal{C}_{0}}^{q} \leq T_{0}^{q} e^{\frac{q \beta h}{2}} \int_{\tau}^{t} e^{\frac{q \beta r}{2}}\left\|u_{r}-v_{r}\right\|_{\mathcal{C}_{0}}^{q} d r, \quad \forall t \geq \tau .
$$

By the Gronwall inequality, we obtain $\left\|u_{t}-v_{t}\right\|=0$, which implies that $\left\|u_{t}-v_{t}\right\|_{\mathcal{C}_{0}}^{2}=0$ for all $t \in[\tau, T]$. Thus, the global solution is unique.

Part (ii): Choose and fix $T>0$ and $\phi, \varphi \in \mathcal{C}_{0}$. Since $u(\cdot, \phi)$ and $v(\cdot, \varphi)$ are solutions of (2.1), condition (H4) implies that

$\|u(t, \phi)-v(t, \varphi)\| \leq e^{-\beta(t-\tau)}\|\phi(0)-\varphi(0)\|+\frac{1}{\Gamma(\alpha)} \int_{\tau}^{t}(t-r)^{\alpha-1} e^{-\beta(t-r)}\left\|u_{r}-v_{r}\right\|_{0} d r$.

Replacing $t$ by $t+\theta$ with $t+\theta \geq \tau$ in the last inequality,

$$
\begin{aligned}
& \|u(t+\theta, \phi)-v(t+\theta, \varphi)\| \\
& \leq e^{-\beta(t+\theta-\tau)}\|\phi-\varphi\|_{\mathcal{C}_{0}}^{q}+T_{0}\left(\int_{\tau}^{t+\theta} e^{-\frac{q \beta(t+\theta-r)}{2}}\left\|u_{r}-v_{r}\right\|_{\mathcal{C}_{0}}^{q} d r\right)^{\frac{1}{q}} .
\end{aligned}
$$

Notice that, for $\tau-h \leq t \leq \tau$, we have

$$
\|u(t, \phi)-v(t, \varphi)\|=\|\phi(t-\tau)-\varphi(t-\tau)\| \leq e^{-\frac{\beta(t+2 \theta-2 \tau)}{2}}\|\phi-\varphi\|_{\mathcal{C}_{0}},
$$

which together with 2.16 yields, for all $t \geq \tau$,

$e^{\frac{q \beta t}{2}}\left\|u_{t}-v_{t}\right\|_{\mathcal{C}_{0}}^{q} \leq 2^{q} e^{\frac{q \beta(t-2 \tau-2 h)}{2}}\|\phi-\varphi\|_{\mathcal{C}_{0}}^{q}+T_{0}^{q} e^{\frac{q \beta h}{2}}\left(\int_{\tau}^{t} e^{\frac{q \beta r}{2}}\left\|u_{r}-v_{r}\right\|_{\mathcal{C}_{0}}^{q} d r\right)$.

By the Gronwall lemma, we conclude, for all $t \geq \tau$,

$$
\left\|u_{t}-v_{t}\right\|_{\mathcal{C}_{0}} \leq 2 e^{\beta h}\|\phi-\varphi\|_{\mathcal{C}_{0}} e^{\left(T_{0}^{q}-\frac{q \beta h}{2}\right) \frac{(t-\tau)}{q}} \rightarrow 0, \text { as } \phi \rightarrow \varphi,
$$

which implies $\lim _{\phi \rightarrow \varphi} \sup \|u(t, \phi)-v(t, \varphi)\|_{\mathcal{C}_{T}}=0$. The proof is completed.

\section{Fractional lattice systems with time variable delays}

Now we will apply our abstract theory developed in the previous section to the fractional lattice system (1.1).

3.1. Setting of the problem. Let $\ell^{2}=\left\{v=\left(v_{i}\right)_{i \in \mathbb{Z}}: \sum_{i \in \mathbb{Z}} v_{i}^{2}<\infty\right\}$ be the separable Hilbert space with norm $\|v\|=\left(\sum_{i \in \mathbb{Z}} v_{i}^{2}\right)^{\frac{1}{2}}$ and scalar product $(w, v)=\sum_{i \in \mathbb{Z}} w_{i} v_{i}$, and let $\ell^{\infty}=\left\{v=\left(v_{i}\right)_{i \in \mathbb{Z}}: \sup _{i \in \mathbb{Z}}\left|v_{i}\right|<\infty\right\}$ be the Banach space with norm $\|v\|_{\infty}=\sup _{i \in \mathbb{Z}}\left|v_{i}\right|$. Further, we use the notation $X=\ell^{2}, \mathcal{C}_{T}=C\left([\tau-h, T] ; \ell^{2}\right)$ and $C_{T, \infty}=C\left([\tau-h, T] ; \ell^{\infty}\right)$, where $T>0$, with the norms

$$
\|u\|_{\mathcal{C}_{T}}=\sup _{s \in[-h, T]}\|u(s)\|, \text { and }\|u\|_{C_{T, \infty}}=\sup _{s \in[-h, T]}\|u(s)\|_{\infty} .
$$


By simple computation, we can verify that $\mathcal{C}_{T} \subset C_{T, \infty}$ with $\|u\|_{C_{T, \infty}} \leq$ $\|u\|_{\mathcal{C}_{T}}$ for all $u \in \mathcal{C}_{T}$.

We consider the system (1.1) on the phase space $\mathcal{C}_{T}$. To this end, we assume the following conditions:

$\left(\mathbf{C}_{\mathbf{1}}\right)$ The operator $f:[\tau, \infty) \times \mathcal{C}_{0} \rightarrow X$ given by the rule $\left(f\left(t, u_{t}\right)\right)_{i}=$ $f_{i}\left(t, u_{i t}\right), i \in \mathbb{Z}$, is well defined, bounded and continuous.

$\left(\mathbf{C}_{2}\right)$ There exists $L_{f}>0$ such that

$$
\|f(t, \phi)-f(t, \varphi)\| \leq L_{f}\|\phi-\varphi\|_{\mathcal{C}_{0}} \quad \text { for all } \phi, \varphi \in \mathcal{C}_{0} \text { and } t \geq \tau .
$$

$\left(\mathbf{C}_{\mathbf{3}}\right)$ The function $f(t, 0)$ is $L^{2}$ local integrable, and there exists a positive constant $L$ such that

$$
\int_{\tau}^{t}(t-r)^{\alpha-1} e^{-\beta(t-r)}\|f(r, 0)\|^{2} d r \leq L, \quad \forall t \geq \tau .
$$

REMARK 3.1. We would like to mention that condition $\left(\mathbf{C}_{\mathbf{1}}\right)$ holds if all the component functions $f_{i}, i \in \mathbb{Z}$, are globally Lipschitz with the same Lipschitz constant, while condition $\left(\mathbf{C}_{\mathbf{2}}\right)$ holds provided that each component function $f_{i}, i \in \mathbb{Z}$, is Lipschitz continuous with a constant $L_{f_{i}}$ and $L_{f}=\left(\sum_{i} L_{f_{i}}^{2}\right)^{1 / 2}<\infty, i \in \mathbb{Z}$. And condition $\left(\mathbf{C}_{\mathbf{3}}\right)$ holds if it holds for each $f_{i}$ with constant $L_{i}$, and $L=\sum_{i} L_{i}<\infty, i \in \mathbb{Z}$.

We first establish the existence of solutions for problem (1.1). For this we rewrite it in abstract form. We define the operator $A: X \rightarrow X$ by

$$
(A v)_{i}=-v_{i-1}+2 v_{i}-v_{i+1}, \quad i \in \mathbb{Z} .
$$

It is easy to check that

$$
\|A v\| \leq 4\|v\| \quad \text { for all } v \in \ell^{2} .
$$

Then the operator $F: \mathcal{C}_{0} \rightarrow X$ is defined by $F(t, v)=-A v(0)-f(t, v)-$ $\lambda v(0)$. Problem (1.1) can be rewritten as

$$
\begin{cases}D_{s}^{\alpha} u(t)=F\left(t, u_{t}\right), & t>\tau \\ u(t)=\phi(t-\tau), & \forall t \in[\tau-h, \tau] .\end{cases}
$$

Theorem 3.1. Assume that $\left(\mathbf{C}_{\mathbf{1}}\right)-\left(\mathbf{C}_{\mathbf{3}}\right)$ hold. Then for every $\phi \in \mathcal{C}_{0}$, the problem 1.1 has a unique solution defined on $[\tau-h, \infty)$.

P r o o f. It is not difficult to check that $F(t, v)$ satisfies $\left(\mathbf{H}_{\mathbf{1}}\right)$ and $\left(\mathbf{H}_{\mathbf{2}}\right)$. Thus the conclusion follows from Theorem 2.2 . 
3.2. Time variable delays. We will consider time variable delays with the delay function satisfying the following condition:

$\left(\mathbf{D}_{1}\right)$ For each $i \in \mathbb{Z}$ the function $\rho_{i}:[0, \infty) \rightarrow[0, h]$ is continuous.

We consider a function $f:[0, \infty) \times \mathcal{C}_{0} \rightarrow X$ given component-wise by the rule $(f(t, v))_{i}=f_{i}\left(t, v_{i}\right)$, where

$$
f_{i}\left(t, v_{i}\right)=F_{1, i}\left(t, v_{i}\left(-\rho_{i}(t)\right)\right)+\int_{-h}^{0} b_{i}\left(t, s, v_{i}(s)\right) d s
$$

with $h>0$, i.e., putting $v=u_{t}=u(t+\cdot)$, problem (1.1) can be rewritten as, for $t>\tau, i \in \mathbb{Z}$,

$$
\left\{\begin{array}{c}
D_{s}^{\alpha} u_{i}(t)-\left(u_{i-1}(t)-2 u_{i}(t)+u_{i+1}(t)\right)+\lambda u_{i}(t)+ \\
F_{1, i}\left(u_{i}\left(t-\rho_{i}(t)\right)\right)+\int_{-h}^{0} b_{i}\left(t, s, u_{i}(t+s)\right) d s=0, \\
u_{i}(t)=\phi_{i}(t-\tau), \forall t \in[\tau-h, \tau] .
\end{array}\right.
$$

We also use the following conditions:

(D) $F_{1, i}$ are continuous in $(t, x)$, and satisfy that $\left|F_{1, i}(t, x)-F_{1, i}(t, y)\right| \leq$ $A_{1}|x-y|$ for all $x, y \in \mathbb{R}$ and uniformly in $t \geq \tau$, where $A_{1}$ is a positive constant and $\left|F_{1, i}(t, 0)\right| \leq\left|A_{2, i}\right|$ and uniformly in $t \geq \tau$, where $A_{2}=\left(A_{2, i}\right)_{i \in \mathbb{Z}} \in \ell^{2}$.

$\left(\mathbf{D}_{3}\right) b_{i}(t, s, x)$ are continuous in $(t, x)$, and $\left|b_{i}(t, s, x)-b_{i}(t, s, y)\right| \leq\left|A_{3, i}\right| \cdot$ $|x-y|$ for all $x, y \in \mathbb{R}$ and a.a. $s \in[-h, 0]$, uniformly in $t>\tau$, and $\left|b_{i}(t, s, 0)\right| \leq m_{0, i}(s)$, with $m_{0, i}(\cdot) \in L^{1}(-h, 0), m_{0, i}(s) \geq 0$, $A_{3}=\left(A_{3, i}\right)_{i \in \mathbb{Z}} \in \ell^{2}$ and $M_{0}^{2}:=\sum_{i \in \mathbb{Z}} M_{0, i}^{2}<\infty$, where $M_{0, i}=$ $\int_{-h}^{0} m_{0, i}(s) d s$.

Let us check conditions $\left(\mathbf{C}_{\mathbf{1}}\right)$ and $\left(\mathbf{C}_{\mathbf{2}}\right)$. First, in order to obtain $\left(\mathbf{C}_{\mathbf{1}}\right)$ we prove that $f$ is well defined and bounded as well as continuous. By $\left(\mathbf{D}_{\mathbf{2}}\right)-\left(\mathbf{D}_{\mathbf{3}}\right)$, we deduce that

$$
\begin{aligned}
& \sum_{i \in \mathbb{Z}}\left|F_{1, i}\left(v_{i}\left(-\rho_{i}(t)\right)\right)\right|^{2} \leq \sum_{i \in \mathbb{Z}} 2 A_{2, i}^{2}+2 A_{1}^{2}\|v\|_{\mathcal{C}_{0}}^{2}, \\
& \sum_{i \in \mathbb{Z}}\left(\int_{-h}^{0}\left|b_{i}\left(t, s, v_{i}(s)\right)\right| d s\right)^{2} \leq 2 h^{2}\left\|A_{3}\right\|^{2}\|v\|_{\mathcal{C}_{0}}^{2}+2 M_{0}^{2},
\end{aligned}
$$

for all $t>\tau$. Then for all $t>\tau$, by $\left(\mathbf{D}_{\mathbf{1}}\right)$, we have

$$
\begin{aligned}
\|f(t, v)\|^{2} & \left.\leq\left. 2\left(\sum_{i \in \mathbb{Z}} \mid F_{1, i}\left(v_{i}\left(-\rho_{i}(t)\right)\right)\right)\right|^{2}+\sum_{i \in \mathbb{Z}}\left(\int_{-h}^{0}\left|b_{i}\left(t, s, v_{i}(s)\right)\right| d s\right)^{2}\right) \\
& \leq 4\left\|A_{2}\right\|^{2}+4 A_{1}^{2}\|v\|_{\mathcal{C}_{0}}^{2}+4 M_{0}^{2}+4 h^{2}\left\|A_{3}\right\|^{2}\|v\|_{\mathcal{C}_{0}}^{2} .
\end{aligned}
$$


And from $\left(\mathbf{D}_{\mathbf{2}}\right)$ and $\left(\mathbf{D}_{\mathbf{3}}\right)$, for any $v, u \in \mathcal{C}_{0}$, we obtain that

$$
\|f(t, v)-f(t, u)\|^{2} \leq\left(2 A_{1}^{2}+2\left\|A_{3}\right\|^{2}\right)\|v-u\|_{\mathcal{C}_{0}}^{2} .
$$

Besides, let $t_{n} \geq \tau,\left\{v_{n}\right\}_{n=1}^{\infty} \subset \mathcal{C}_{0}$, and $t \geq \tau, v \in \mathcal{C}_{0}$ such that $t_{n} \rightarrow t$, and $v_{n} \rightarrow v$ in $\mathcal{C}_{0}$. Notice that $F_{1, i}$ and $b_{i}(t, s, x)$ are continuous in $(t, x)$, we have when $n$ goes to infinity

$$
\begin{aligned}
& \left\|f_{i}\left(t_{n}, v_{n}\right)-f_{i}(t, v)\right\| \\
& \leq\left\|F_{1, i}\left(t_{n}, v_{n}\right)-F_{1, i}(t, v)\right\|+\left\|\int_{-h}^{0} b_{i}\left(t_{n}, s, v_{n}(s)\right)-b_{i}(t, s, v(s)) d s\right\| \rightarrow 0 .
\end{aligned}
$$

Therefore $\left(\mathbf{C}_{\mathbf{1}}\right)$ holds true.

On the other hand, for any $t>\tau$,

$$
\begin{aligned}
& \int_{\tau}^{t}(t-r)^{\alpha-1} e^{-\beta(t-r)}\|f(r, 0)\|^{2} d r \\
& =\int_{\tau}^{t}(t-r)^{\alpha-1} e^{-\beta(t-r)} \sum_{i \in \mathbb{Z}}\left|F_{i}(r, 0)+\int_{-h}^{0} b_{i}(r, s, 0) d s\right|^{2} d r \\
& \leq 2 \beta^{-\alpha}\left(\left\|A_{2}\right\|^{2}+M_{0}^{2}\right)<\infty .
\end{aligned}
$$

Note that the $F_{1, i}$ are Lipschitz continuous and the $b_{i}(t, s, x)$ are Lipschitz continuous in $x$. Hence, $\left(\mathbf{C}_{\mathbf{1}}\right),\left(\mathbf{C}_{\mathbf{2}}\right)$ and $\left(\mathbf{C}_{\mathbf{3}}\right)$ are true by $(3.3)-(3.5)$. Thanks to Theorem 3.1, we see that for every $\phi \in \mathcal{C}_{0}$, problem 3.2 has a unique solution defined on $[\tau-h, \infty)$.

\section{Estimates of solutions}

We obtain some estimates of solutions in this section. Such estimates will imply that the solutions are bounded uniformly with respect to bounded sets of initial conditions and positive values of time.

Lemma 4.1. Assume that $\left(\mathbf{D}_{\mathbf{1}}\right),\left(\mathbf{D}_{\mathbf{2}}\right)$ and $\left(\mathbf{D}_{\mathbf{3}}\right)$ hold and there exist positive constants $q$ and $c$ such that

$$
q \beta-2 c>0 .
$$

Then, every solution $u(\cdot)$ of $(3.2)$ with $u_{\tau}=\phi \in \mathcal{C}_{0}$ verifies

$$
\begin{gathered}
\left\|u_{t}\right\|_{\mathcal{C}_{0}}^{2 q} \leq \frac{2 a c}{3 q \beta+c} e^{-\left(\frac{q \beta}{2}-c\right)(t-\tau)}+a+b+\frac{2 b c}{q \beta-2 c}, \\
\text { where } a=3^{q-1} 2^{q} e^{2 q \beta h}\|\phi\|_{\mathcal{C}_{0}}^{2 q}, b=3^{q-1} C_{1}^{q}, c=C_{2}^{q}\left(\frac{\Gamma^{\frac{1}{1+\alpha}}\left(\alpha^{2}\right)}{\left(\frac{p \beta}{2}\right)^{\frac{\alpha^{2}}{1+\alpha}}}\right)^{q} e^{\frac{q \beta h}{2}} .
\end{gathered}
$$


P r o o f. The proof is similar to those of Lemma 2.3 and Theorem 2.2.

For the proof of compactness of the attracting set, we need the following lemma.

Lemma 4.2. Suppose that assumptions of Lemma 4.1 hold, and there exists a positive constant $C_{8}$ such that $q \beta>2 C_{8}$. Then, for any $\varepsilon>0$ there exist $T(\varepsilon, B), K(\varepsilon, B)$ such that

$$
\sup _{\theta \in[-h, 0]} \sqrt{\sum_{|i| \geq 2 K(\varepsilon, B)}\left|u_{i}(t+\theta)\right|^{2}}<\tilde{C} \varepsilon, \quad t \geq T(\varepsilon, B),
$$

for any initial condition $\phi \in B \subset \mathcal{C}_{0}$ and any solution $u(\cdot)$ of 3.2 with $u_{\tau}=\phi$.

P r o o f. The proof is similar to that in Bates et al. [1], so is omitted.

\section{Main results}

\subsection{Existence of the global attracting set.}

Theorem 5.1. Assume that $q \beta>2 \max \left\{c, C_{8}\right\}$. Then

(1) for any bounded subset $B \subset \mathcal{C}_{T}$, any sequence $\left\{t_{n}\right\}$ with $t_{n} \rightarrow$ $\infty(n \rightarrow \infty),\left\{\phi^{n}\right\}$ with $\phi^{n} \in B$, any sequence of solutions $\left\{u^{n}(\cdot)\right\}$ of problem (3.2) with $u_{\tau}^{n}=\phi^{n} \in B$, the sequence $\left\{u_{t_{n}}^{n}\right\}$ is relatively compact in $\mathcal{C}_{T}$;

(2) for any bounded subset $B$ of $\mathcal{C}_{T}$, the set

$\omega(B)=\left\{\psi: \exists t_{n} \rightarrow \infty, \phi^{n} \in B\right.$ and a sequence of solutions $u^{n}(\cdot)$ of problem 3.2 with $u_{\tau}^{n}=\phi^{n} \in B$ such that $u_{t_{n}}^{n} \rightarrow \psi$ in $\left.\mathcal{C}_{T}\right\}$

is nonempty, compact and attracts $B$;

(3) the set $A=\overline{\bigcup\left\{\omega(B): B \subset \mathcal{C}_{T}, B \text { bounded }\right\}}$ is bounded in $\mathcal{C}_{T}$, compact in the topology of $C_{0}$. Moreover, it is the minimal closed set that attracts all bounded subsets of $\mathcal{C}_{T}$ in the topology of $\mathcal{C}_{T}$, where $\bar{E}$ denotes the closure of $E$.

P r o o f. The proof is based on [1] and is given in four steps:

Step 1. Without loss of generality, we assume that $t_{n} \geq h$ for all $n \in \mathbb{N}$ and $\|\phi\|_{\mathcal{C}_{T}} \leq d$ for all $\phi \in B$. Thanks to Lemma 4.1, for fixed $\theta \in[-h, 0]$ we can find a subsequence (denoted as $u^{n}$ ) such that $u_{t_{n}}^{n}(\theta) \rightarrow v$ in $\ell^{2}$. In 
fact, the convergence is strong, which can be deduced from Lemma 4.2 . Indeed, for any $\varepsilon>0$ there exist $M(\varepsilon)$ and $N(\varepsilon)$ such that

$$
\sup _{\theta \in[-h, 0]} \sqrt{\sum_{|i| \geq 2 M}\left|u_{i}^{n}\left(t_{n}+\theta\right)\right|^{2}}<\varepsilon, \quad \sqrt{\sum_{|i| \geq 2 M}\left|v_{i}\right|^{2}}<\varepsilon,
$$

and $\sqrt{\sum_{|i| \leq 2 M}\left|u_{i}^{n}\left(t_{n}+\theta\right)-v_{i}\right|^{2}}<\varepsilon$ if $n \geq N$, so that

$$
\left\|u_{t_{n}}^{n}(\theta)-v\right\| \leq \sqrt{\sum_{|i|>2 M}\left|u_{i}^{n}\left(t_{n}+\theta\right)-v_{i}\right|^{2}}+\sqrt{\sum_{|i| \leq 2 M}\left|u_{i}^{n}\left(t_{n}+\theta\right)-v_{i}\right|^{2}}<C \varepsilon .
$$

Thus, $\left\{u_{t_{n}}^{n}(\theta)\right\}$ is precompact in $\ell^{2}$ for any $\theta \in[-h, 0]$. In order to apply the Arzelà-Ascoli theorem, we need to check the equi-continuity property. To do this, by Eq.(3.1), (D2)-(D3) and Lemma 4.1, we obtain that every solution $u(\cdot)$ of $(3.2)$ with $u_{\tau}=\phi \in B \subset \mathcal{C}_{T}$ satisfies

$$
\begin{aligned}
& \left.\|A u(t)\|+|\lambda|\|u(t)\|+\| F_{1}\left(u\left(t-\rho_{i}(t)\right)\right)\right)\|+\| \int_{-h}^{0} b(t, \theta, u(t+\theta)) d \theta \| \\
& \leq(4+|\lambda|)\|u(t)\|+\sqrt{2\left\|A_{2}\right\|^{2}+2 A_{1}^{2}\left\|u_{t}\right\|_{\mathcal{C}_{T}}^{2}}+\sqrt{2 M_{0}^{2}+2 M_{1}^{2}\left\|u_{t}\right\|_{\mathcal{C}_{T}}^{2}} \leq c^{\prime},
\end{aligned}
$$

for all $t \geq \tau$. Arguing as in the proof of Theorem 2.2, we have that for $n \in \mathbb{N}$ and $\theta_{1}, \theta_{2} \in[-h, 0]$,

$$
\left\|u^{n}\left(t_{n}+\theta_{1}\right)-u^{n}\left(t_{n}+\theta_{2}\right)\right\| \leq c^{\prime}\left|e^{-\beta \theta_{1}}-e^{-\beta \theta_{2}}\right|+c^{\prime}\left|\theta_{1}-\theta_{2}\right|^{\alpha} .
$$

Then, Arzelà-Ascoli's theorem implies that $u_{t_{n}}^{n}$ is relatively compact in $\mathcal{C}_{T}$.

Step 2. By the conclusion (1) and the definition of $\omega(B)$, the nonemptiness and compactness of $\omega(B)$ follow immediately. Now we show that $\omega(B)$ attracts $B$. If not, there exist $\varepsilon^{\prime}>0$ and sequences $\left\{t_{n}\right\}$ with $t_{n} \rightarrow \infty,\left\{\phi^{n}\right\}$ with $\phi^{n} \in B$ and solutions $\left\{u^{n}(\cdot)\right\}$ of $(3.2)$ with $u_{0}^{n}=\phi^{n}$ such that

$$
\operatorname{dist}_{\mathcal{C}_{T}}\left(u_{t_{n}}^{n}, \omega(B)\right)>\varepsilon^{\prime}, \quad \forall n \in \mathbb{N},
$$

where $\operatorname{dist}_{\mathcal{C}_{T}}(\cdot, \cdot)$ is the norm of $\mathcal{C}_{T}$. Using the conclusion (1), we obtain that $u_{t_{n}}^{n}$ is relatively compact and possesses at least one cluster point $v$ in $\mathcal{C}_{T}$. By the definition of $\omega(B)$, it is clear that $v \in \omega(B)$, and this contradicts (5.1).

Step 3. Let $\widetilde{A}=\bigcup\left\{\omega(B): B \subset \mathcal{C}_{T}, B\right.$ bounded $\}$, and $\left\{y_{n}\right\}_{n=1}^{\infty}$ be a sequence in $\widetilde{A}$ with $y_{n} \in \omega\left(B_{n}\right)$ and $\left\|B_{n}\right\|_{\mathcal{C}_{T}}=\sup _{\psi \in B_{n}}\|\psi\|_{\mathcal{C}_{T}} \leq d_{n}$. Then by the definition of $\omega\left(B_{n}\right)$, we can deduce that there exist sequences $\left\{t_{n}\right\}$ with $t_{n} \rightarrow \infty$ with $\phi^{n} \in B_{n}$, and solutions $\left\{u_{t_{n}}^{n}(\cdot)\right\}$ of $(3.2)$ with $u_{\tau}^{n}=\phi^{n} \in B_{n}$ such that for all $n \in \mathbb{N}$,

$$
\left\|u_{t_{n}}^{n}-y_{n}\right\|_{C_{0}} \leq \frac{1}{n}
$$


Now we need to prove that $u_{t_{n}}^{n}$ is relatively compact in $C_{0, w}$. Since $X=\ell^{2}$ is reflexive, from Lemma 4.1 we see that for fixed $\theta \in[-h, 0],\left\{u_{t_{n}}^{n}(\theta)\right\}$ is relatively compact in $X_{w}$. On the other hand, we deduce that $\left\{u_{t_{n}}^{n}\right\}$ is equicontinuous. In fact, by the method we used in Step 1, we could obtain that from Lemma 2.2. $\left(\mathbf{C}_{\mathbf{5}}\right)$ and $\left(\mathbf{C}_{\mathbf{6}}\right)$, if $n \in \mathbb{N}, \theta_{1}, \theta_{2} \in[-h, 0]$ and $\theta_{1} \leq \theta_{2}$, then we have for all $n$ sufficiently large,

$$
\left\|u^{n}\left(t_{n}+\theta_{1}\right)-u^{n}\left(t_{n}+\theta_{2}\right)\right\| \leq C\left|e^{-\beta \theta_{1}}-e^{-\beta \theta_{2}}\right|+C\left(\theta_{2}-\theta_{1}\right)^{\alpha} .
$$

Thus we conclude that $\left\{u_{t_{n}}^{n}\right\}$ is relatively compact in $C_{0}$. Combining this with $(5.2)$, we obtain that $\left\{u^{n}(\cdot)\right\}$ is relatively compact in $C_{0}$, and thus

$$
A=\overline{\bigcup\left\{\omega(B): B \subset \mathcal{C}_{T}, B \text { bounded }\right\}}
$$

is compact in the topology of $C_{0}$.

From Lemma 4.1 and the conclusion (2), we see that $A$ is bounded in $\mathcal{C}_{T}$, and attracts all bounded subsets of $\mathcal{C}_{T}$ in the topology of $\mathcal{C}_{T}$.

Finally, we show that $A$ is the minimal closed set attracting set. Indeed, if there is another closed set $A^{\prime}$ which attracts any bounded set $B \subset \mathcal{C}_{T}$, then by the definition of $\omega(B)$, we deduce that $\omega(B) \subset A^{\prime}$, and thus $\bigcup\left\{\omega(B): B \subset \mathcal{C}_{T}, B\right.$ bounded $\}$ belongs to $A^{\prime}$. Since $A^{\prime}$ is closed, we have $A=\overline{\bigcup\left\{\omega(B): B \subset \mathcal{C}_{T}, B \text { bounded }\right\}} \subseteq A^{\prime}$. The proof of Theorem 5.1 is completed.

5.2. Singleton case. To show that the forward attracting set is a singleton set, we need the following lemma, which is proved like Theorem 2.2 .

Lemma 5.1. Assume that

$$
e^{-\frac{\beta h}{2}} \beta^{2 \alpha}>2^{\alpha+\frac{1}{1+\alpha}} p^{-\frac{\alpha^{2}}{1+\alpha}} q^{-\frac{\alpha}{1+\alpha}} \Gamma^{\frac{1}{1+\alpha}}\left(\alpha^{2}\right) C_{11},
$$

where $C_{11}:=\frac{8\left(16+\lambda^{2}+A_{1}^{2}+M_{1}^{2}\right)}{\Gamma(\alpha)}$.

Then for any two solutions $u(t)$ and $w(t)$ of problem (3.2) corresponding to initial values $\phi$ and $\psi$, we have the strict contracting property

$$
\left\|u_{t}-w_{t}\right\|_{\mathcal{C}_{T}}^{2 q} \leq C\|\phi-\psi\|_{\mathcal{C}_{T}}^{2 q} e^{-\left(\frac{q \beta}{2}-C_{12}\right)(t-\tau)} \quad \forall t \geq \tau
$$

where $C_{12}:=2^{\alpha+\frac{1}{\alpha}} p^{-\alpha} \Gamma^{\frac{1}{\alpha}}\left(\alpha^{2}\right) C_{11}^{q} e^{\frac{q \beta h}{2}} \beta^{-2 \alpha}$.

Theorem 5.2. Assume the condition in Theorem5.1. If we additionally assume that, for each $i \in \mathbb{Z}$ and any $s \in[-h, 0], F_{1, i}(0)=b_{i}(t, s, 0)=0$. Then the set $A=\bigcup\left\{\omega(B): B \subset \mathcal{C}_{T}, B\right.$ bounded $\}$ is a singleton set. Moreover, it is the minimal set that exponentially attracts all bounded subsets of $\mathcal{C}_{T}$. Thus, every solution exponentially decays to zero in the topology of $\mathcal{C}_{T}$. 
P r o o f. Since for each $i \in \mathbb{Z}$ and any $\theta \in[-h, 0], F_{1, i}(0)=b_{i}(t, \theta, 0)=$ 0 , it follows from Lemma 4.1 that for any solution $u(\cdot)$ of problem $(3.2)$ with initial value $\phi$, we have

$$
\left\|u_{t}\right\|_{\mathcal{C}_{T}}^{2 q} \leq C\|\phi\|_{\mathcal{C}_{0}}^{2 q} e^{-\left(\frac{q \beta}{2}-c\right) t} \quad, \forall t \geq 0
$$

Let $B$ be a bounded subset of $\mathcal{C}_{T}$ with $\|B\|_{\mathcal{C}_{T}}=\sup _{\psi \in B}\|\psi\|_{\mathcal{C}_{T}} \leq d$.

We show that $\omega(B)$ is a singleton set. If not, then there would exist $x, y \in \omega(B)$ such that $x \neq y$. By the definition of $\omega(B)$, there exist sequences $\left\{\tau_{n}\right\}$ and $\left\{s_{m}\right\}$, respectively, with $\tau_{n} \rightarrow \infty(n \rightarrow \infty)$ and $s_{m} \rightarrow \infty$ $(m \rightarrow \infty),\left\{\phi^{n}\right\}$ and $\left\{\psi^{m}\right\}$ with $\phi^{n}, \psi^{m} \in B$, solutions $\left\{u^{n}(\cdot)\right\}$ and $\left\{w^{m}(\cdot)\right\}$ of problem (3.2) with $u_{\tau}^{n}=\phi^{n}, w_{\tau}^{m}=\psi^{m}$ such that

$$
u_{\tau_{n}}^{n} \rightarrow x \quad(n \rightarrow \infty) \quad \text { and } \quad w_{s_{m}}^{m} \rightarrow y \quad(m \rightarrow \infty) \quad \text { in } \mathcal{C}_{T} .
$$

From 5.3 we deduce that

$$
\left\|u_{\tau_{n}}^{n}-w_{s_{m}}^{m}\right\|_{\mathcal{C}_{T}} \leq C e^{-\frac{\left(q \beta-2 C_{12}\right)}{2 q}\left(\tau_{n}-\tau\right)}+C e^{-\frac{(q \beta-2 c)}{2 q}\left(s_{m}-\tau\right)},
$$

which implies that

$$
\left\|u_{\tau_{n}}^{n}-w_{s_{m}}^{m}\right\|_{\mathcal{C}_{T}} \rightarrow 0 \quad \text { as } n \rightarrow \infty \text { and } m \rightarrow \infty
$$

Hence, $\|x-y\|_{\mathcal{C}_{T}}=0$, this is a contradiction. Since $\omega(B)$ is the same singleton set for each bounded set $B$, we obtain from (5.3) that $A$ is a singleton set. Indeed, as $\omega\left(B_{1}\right)=\left\{x_{1}\right\}$ and $\omega\left(B_{2}\right)=\left\{x_{2}\right\}$. Now $B=$ $B_{1} \cup B_{2}$, so $\omega\left(B_{i}\right) \subset \omega(B)$ for $i=1,2$, but as $\omega(B)$ only contains one point. Hence $x_{1}=x_{2}$. Moreover, every solution exponentially decays to zero in the topology of $\mathcal{C}_{T}$. Thus the proof is completed.

\section{References}

[1] P. Bates, K. Lu and B. Wang, Attractors for lattice dynamical systems. Int. J. Bifur. Chaos 11 (2001), 143-153.

[2] T. Caraballo, F. Morillas and J. Valero, On differential equations with delay in Banach spaces and attractors for retarded lattice dynamical systems. Discrete Contin. Dyn. Syst. 34 (2014), 51-77.

[3] S. Carmi and E. Barkai, Fractional Feynman-Kac equation for weak ergodicity breaking. Phys. Rev. E Statistical Nonlinear Soft Matter Phys. 84 (2011), Art. \# 061104.

[4] P. Carr, H. Geman, D.B. Madan and M. Yor, Stochastic volatility for Lévy processes. Math. Finance 13 (2003), 345-382.

[5] M. Chen and W. Deng, Discretized fractional substantial calculus. ESAIM Math. Model. Numer. Anal. 49 (2015), 373-394.

[6] M. Chen and W. Deng, High order algorithms for the fractional substantial diffusion equation with truncated Lévy flights. SIAM J. Sci. Comput. 37 (2015), A890-A917. 
[7] W. Deng, M. Chen and E. Barkai, Numerical algorithms for the forward and backward fractional Feynman-Kac equations. J. Sci. Comput. 62 (2015), 718-746.

[8] R. Friedrich, F. Jenko, A. Baule and S. Eule, Anomalous diffusion of inertial, weakly damped particles. Phys. Rev. Lett. 96 (2006), Art. \# 230601.

[9] A. Granas, J. Dugundji, Fixed Point Theory, Springer Monographs in Mathematics. Springer, New York (2003).

[10] A.A. Kilbas, H.M. Srivastava and J.J. Trujillo, Theory and Applications of Fractional Differential Equations. Elsevier Science B.V., Amsterdam (2006).

[11] M. Meerschaert, Y. Zhang and B. Baeumer, Tempered anomalous diffusions in heterogeneous systems. Geophys. Res. Lett. 35 (2008), L17403-L17407.

[12] D. O'Regan, Fixed point theorems for nonlinear operators, J. Math. Anal. Appl. 202 (1996), 413-432.

[13] M. Zayernouri, M. Ainsworth and G. Karniadakis, Tempered fractional Sturm-Liouville eigenproblems. SIAM J. Sci. Comput. 37 (2015), A1777-A1800.

1 Dept. Applied Mathematics, School of Mathematics and Statistics

Xi'an Jiaotong Univeristy,

Xianning West Road 28, 710049, Xi'an, Shaanxi, P.R. CHINA

e-mail: liulinfang1988@163.com

${ }^{2}$ Dpto. Ecuaciones Diferenciales y Análisis Numérico

Facultad de Matemáticas, Universidad de Sevilla

c/Tarfia s/n, 41012 Sevilla, SPAIN

e-mail: caraball@us.es

3 Dpto. Mathematisches Institut, Universität Tübingen

D-72076 Tübingen, GERMANY

e-mail: kloeden@math.uni-frankfurt.de_Received: December 13, 2018 